\title{
Structural peculiarities and polymorphism of the SQS-gene controlling the synthesis of squalene in amaranth
}

\author{
Shcherban A.B.*, Stasyuk A.I., Salina E.A. \\ Institute of Cytology and Genetics, SB RAS, Novosibirsk, Russia \\ *e-mail:atos@bionet.nsc.ru
}

Amaranth (genus Amaranthus) is a unique dicotyledonous plant with great potential as a grain, vegetable and fodder crop. Oil from amaranth grain is enriched with valuable lipid compounds, in particular, squalene, the content of which (2.2-10\%) significantly exceeds the content of squalene in other plant oils [1]. Squalene is widely used in medicine: as an adjuvant in vaccines, immunomodulator and antioxidant in the complex therapy of a such diseases as diabetes, ischemic disease, etc., as part of cosmetics [2]. The key gene in squalene biosynthesis is the gene encoding the enzyme squalenesynthase (SQS), which catalyzes the final stage of the formation of a squalene molecule. The aim of our work is to study the polymorphism of $S Q S$-gene in different species of Amaranthus, as well as to search for those features in its structure that may determine the variation of squalene concentration in amaranth tissues. On the basis of the reference genomic sequence of A. hypochondriacus, available in the GoGe database (id40120; https://genomevolution.org/coge/) we have established a nucleotide sequence of the $S Q S$-gene and designed specific primers for the promoter ( $\sim 1 \mathrm{kbp}$ upstream ATG-codon) and coding regions of this gene. As the latter, a region between exons 5 and 9 was taken, containing 3 functionally conservative domains. Using these primers, isolation and analysis of primary structure of the $S Q S$-gene in various species, the representatives of the "grain" amaranth (A. hypochondriacus, A. cruentus, A. caudatus) and their wild predecessors was carried out. The association of revealed structural polymorphism of $S Q S$-gene with squalene concentration in amaranth grain tissue was analyzed.

Acknowledgements: This work were supported by project No. 0324-2019-0039.

\section{References}

1 Zheleznov A.V., Zheleznova N.B., Burmakina N. In Yudina R.S. Amaranth: scientific bases of introduction. Novosibirsk: Acad. Publ. House "Geo", 2009.

2 Huang Z.R., Lin Y.K., Fang J.Y., Biological and pharmacological activities of squalene and related compounds: potential uses in cosmetic dermatology. Molecules. 2009:14;540-544. 